\title{
Determination of the averaged charge-to-mass ratio of the heavy charged constituents of a magnetoplasma using whistler wave measurements
}

\author{
C. Krafft ${ }^{1,2}$ and B. V. Lundin ${ }^{3}$ \\ ${ }^{1}$ Laboratoire de Physique des Plasmas, Ecole Polytechnique, UMR 7648, 91128 Palaiseau Cedex, France \\ ${ }^{2}$ Paris South University, 91405 Orsay Cedex, France \\ ${ }^{3}$ Pushkov Institute of Terrestrial Magnetism, Ionosphere and Radiowave Propagation, Russian Academy of Sciences, Troitsk, \\ Moscow Region, 142190, Russia
}

Received: 2 April 2010 - Revised: 13 September 2010 - Accepted: 11 December 2010 - Published: 23 December 2010

\begin{abstract}
In a cold magnetized plasma with two light ions of comparable gyrofrequencies and any species of heavy ions and/or charged dust particulates, a technique is developed to recover the relative charge density of the heavy plasma population and to estimate its effective averaged charge-to-mass ratio. Such results can be obtained without using mass spectrometer data but only the measurements of the ion plasma frequency, the electron gyro- and plasma frequencies as well as the two highest ion cutoff frequencies.
\end{abstract}

Keywords. Ionosphere (Wave propagation) - Radio science (Waves in plasma) - Space plasma physics (Waves and instabilities)

\section{Introduction}

An effective approach to determine numerically reliable approximations of the electron whistler dispersion law in cold magnetized plasmas of moderate densities containing several ion species of both charge signs was demonstrated recently (Lundin and Krafft, 2008, 2009a, b). To realize it under typical conditions of laboratory and space plasmas, it is sufficient to provide, using data registered by wideband electromagnetic wave receivers, the values of the characteristic frequencies of the plasma in three distant frequency domains, which cover the ranges of the electron gyro- and plasma frequencies ( $\omega_{\mathrm{c}}$ and $\omega_{\text {pe }}$, respectively), the domain of the ion plasma and/or lower hybrid resonance (LHR) frequencies as well as the ultra-low frequency region near the lightest ions' gyrofrequencies. Actually, in moderate density plasmas (when

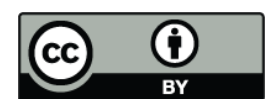

Correspondence to: $\mathrm{C}$. Krafft (catherine.krafft@lpp.polytechnique.fr) $\omega_{\text {pe }} \sim \omega_{\mathrm{c}}$ ), the relative charge densities of the two lightest ion species can be recovered if the much more heavy ion varieties or charged dust particulates can be considered, under the action of electron whistler electromagnetic fields, as a quasi-immobile plasma background (so-called IPB, Lundin and Krafft, 2009a) with constituents of very small gyrofrequencies which contribute mainly to the plasma charge neutrality condition. The present paper is aimed at finding the conditions for which an effective averaged gyrofrequency (or averaged charge-to-mass ratio) of all the heavy ion species or charged dust particulates forming the IPB can be estimated.

The contributions of ions to the dispersive features of electromagnetic waves propagating in multi-ion plasmas was discussed by numerous authors (e.g. Hines, 1957; Buchsbaum, 1960; Yakimenko, 1962; Gintzburg, 1963; Smith and Brice, 1964; Smith, 1965; Gurnett and Burns, 1968; Muzzio, 1968; Das and Uberoi, 1972) in application to laboratory and space plasma investigations (see also Gurnett and Bhattacharjee, 2005, and references therein). At the same time, various diagnostic approaches have been suggested and applied to estimate the ion composition in space plasmas using the registration of the so-called ion cyclotron whistler spectrograms, taking into account that several plasma characteristic frequencies (as the lower hybrid resonance frequency $\omega_{\mathrm{LH}}$, the ion cutoff and resonance frequencies, the so-called crossover frequencies) together with the charge neutrality condition can be expressed through the same ion composition parameters (Shawhan, 1966a, b; Shawhan and Gurnett, 1966; Gurnett and Bhattacharjee, 2005; Rauch et al., 2010). In this view, a new approach to estimate the ion composition was presented recently by the authors (Lundin and Krafft, 2009a). It is based on simplified but numerically reliable dispersion equations for the electron whistler waves and the adjacent in frequency ion cyclotron whistlers, applicable in plasmas

Published by Copernicus Publications on behalf of the European Geosciences Union. 
with two different groups of charged particles: two light ion species of comparable gyrofrequencies and a charged plasma background of quasi-immobile heavy ions and/or charged dust particulates, whose contributions mainly affect the quasi-neutrality condition. At the same time, it was shown that the presence of negatively charged light ions and heavy plasma particulates (and/or charged dust belonging to the IPB) can essentially increase the ion cutoff frequencies for the electron whistlers, as well as lead to the merging of the cutoff frequencies of the neighbor wave branches, i.e. the electron whistler and the ion cyclotron whistler modes.

Complex mixtures of heavy ions of different origins, nanoparticles and charged dust grains of wide ranges of charge-to-mass ratios have been observed in the interplanetary space, in the ionospheres and the magnetospheres of giant planets and their satellites as well as in the surrounding of comets (e.g. Goertz, 1989; Young et al., 2005; Cravens et al., 2006, 2009; Coates et al., 2009; Meyer-Vernet et al., 2009; Mann et al., 2009; Sittler et al., 2009; Wahlund et al., 2009, and references therein). In particular, fast streams of charged particulates encountering the surface of space vehicles produce transient voltage effects in the registered electric fields. In turn, we show here that some properties of the charged dust can be investigated not only by mass spectrometer tools but also through its contribution to the dispersive properties of electron whistler waves of low frequencies lying near the lightest ions' gyrofrequencies; in this case, the ion composition parameters are actually averaged over a space region of the order of several whistler wavelengths, exceeding essentially any vehicle size.

The developed approach can be applied to characterize the heavy particle populations of dusty magnetized plasmas as those encountered in the ionized surroundings of Saturn, Enceladus or Titan, for example, when the full necessary set of characteristic plasma frequencies will be available. Indeed, for the latter case, mass spectrometer data indicate the existence of a very complex plasma chemistry and, notably, the presence of many species of negative and positive charged ions (e.g. Cravens et al., 2006; Coates et al., 2009; Sittler et al., 2009; Wahlund et al., 2009). In particular, at the height of $950 \mathrm{~km}$ in Titan's ionosphere, very heavy negative ions have been detected among which several families of mass-per-charge ratios can be identified, corresponding to "light" ions (10-20 amu q ${ }^{-1}$ ) and to much more heavier ones (up to $100-200 \mathrm{amu} \mathrm{q}^{-1}$ and more). Moreover, the presence of energetic electron and ion fluxes and beams have been observed in such plasmas; they can excite low frequency electron whistler waves through various instability mechanisms (e.g. Krafft and Volokitin, 2003, 2006; Krafft et al., 2005, and references therein). Then, in such ionized surroundings involving mixtures of rather light ions with heavier particle populations, the presented approach could be applied successfully and lead to quantitative results when all necessary in situ whistler measurements will be available.
Developing the approach presented in our recent works (Lundin and Krafft, 2008, 2009a, b), we analyze in this paper the equations for the ion cutoff frequencies considering cold multi-ion magnetized plasmas of moderate densities consisting in two groups of charged particles: (i) two light ions of comparable gyrofrequencies and (ii) a significantly more heavy population of ions and/or charged dust of different charge signs. Our aim is to determine the physical conditions for which the averaged charge-to-mass ratio and the effective averaged gyrofrequency of the most heavy constituents of the plasma background can be estimated. Moreover we propose a method to determine more accurately the value of $\omega_{\mathrm{LH}}$ (or the ion plasma frequency $\omega_{\text {pi }}$, so that the typical spread of the intense line visible on the registered spectrograms near the local LHR frequency can not induce inaccurate estimates of the background plasma ion composition. Actually improved estimates of $\omega_{\mathrm{pi}}$ can be reached (compared to previous studies) if the wave magnetic field polarization data are available, then in moderate density plasmas our method is based on the fast variation with frequency of the plane wave polarization ellipses near $\omega_{\mathrm{pi}}$.

\section{Equation for the ion cutoff frequencies}

Let us find the physical conditions for which it is possible to determine an effective averaged gyrofrequency of the Immobile Plasma Background (IPB) and thus to obtain an additional characteristic parameter of the plasma. We will proceed in a similar way as in our previous works (Lundin and Krafft, 2008, 2009a, b), where the roots of the equation $F(\omega)=\left(\varepsilon^{2}-g^{2}\right) / \eta=0$, corresponding to the two highest ion cutoff frequencies, and the charge neutrality condition have been used jointly to determine the relative charge densities of the two lightest ion species and of all the other heavy charged particulates constituting the IPB as a whole. The parameters $\varepsilon, g$ and $\eta$ are the plasma permittivity tensor components defined according to the conventional notations of Shafranov (1967). Considering the equation $F(\omega)=$ $\left(\varepsilon^{2}-g^{2}\right) / \eta=0$, we can express the terms $\varepsilon \pm g$ as

$\varepsilon \pm g=1 \pm \frac{n}{n_{\mathrm{e}}} \frac{\omega_{\mathrm{pe}}^{2}}{\omega \omega_{\mathrm{c}}}\left(\frac{v_{\mathrm{e}} \omega}{\omega \pm \omega_{\mathrm{c}}}+\sum_{\beta} \frac{v_{\beta} \omega}{\omega \pm \omega_{\beta}}-\sum_{\alpha} \frac{v_{\alpha} \omega}{\omega \mp \omega_{\alpha}}\right)$,

where $v_{j} \equiv Z_{j} n_{j} / n>0$ and $\omega_{j}=Z_{j} e B_{0} / M_{j} c>0$ are the relative charge density and the gyrofrequency of the ion species $j=\alpha, \beta ; M_{j}, n_{j}$ and $Z_{j}>0$ are the corresponding particle mass, density and charge number; $e>0$ is the electron charge; $B_{0}$ is the intensity of the ambient magnetic field; $c$ is the speed of light; the index $\alpha$ corresponds to the positively charged particles, with a total charge density $n$ (expressed in electron charge units), and $\beta$ to the negatively charged ones; $\omega_{\mathrm{pe}}, n_{\mathrm{e}}$, and $v_{\mathrm{e}}=n_{\mathrm{e}} / n$ are the electron plasma frequency, the electron density and its normalized value, respectively; we use the notations $\omega_{\mathrm{H}}$ and $\omega_{\mathrm{c}}$ for the proton and the electron gyrofrequencies. 
Actually, considering a plasma with two positively charged ion species - labeled by indices " 1 " and " 2 " in the following equations - and a charged IPB, Eq. (1) can be written in the form

$$
\begin{aligned}
& \varepsilon-g \simeq 1+\frac{n}{n_{\mathrm{e}}} \frac{\omega_{\mathrm{pe}}^{2}}{\omega \omega_{\mathrm{c}}}\left(v_{\mathrm{e}}-\frac{v_{1} \omega_{1}}{\omega+\omega_{1}}-\frac{v_{2} \omega_{2}}{\omega+\omega_{2}}-\sum_{\mathrm{IPB}} \frac{v_{j} \omega_{j}}{\omega}\right), \\
& \varepsilon+g \simeq 1-\frac{n}{n_{\mathrm{e}}} \frac{\omega_{\mathrm{pe}}^{2}}{\omega \omega_{\mathrm{c}}}\left(v_{\mathrm{e}}+\frac{v_{1} \omega_{1}}{\omega-\omega_{1}}+\frac{v_{2} \omega_{2}}{\omega-\omega_{2}}+\sum_{\mathrm{IPB}} \frac{v_{j} \omega_{j}}{\omega}\right),
\end{aligned}
$$

where the small parameters $\omega / \omega_{\mathrm{c}} \ll 1$ and $\omega_{j} / \omega \ll 1(j \neq$ $1,2)$ have been neglected. We took into account that the relative charge densities of the particles verify the charge neutrality condition

$$
1=\sum_{\alpha} v_{\alpha}=v_{\mathrm{e}}+\sum_{\beta} v_{\beta}
$$

As the plasma includes negative ions we also use the notation $\omega_{\mathrm{p}}$ for the electron plasma frequency calculated for an electron density $n_{\mathrm{e}}$ equal to the total density $n$ of the positive charges, i.e. $\omega_{\mathrm{pe}}^{2}=v_{\mathrm{e}} \omega_{\mathrm{p}}^{2}$ (see also the Appendix B for the definition of the ion plasma frequency $\omega_{\mathrm{pi}}$ so that $\omega_{\mathrm{LH}}^{2} \equiv$ $\mu \omega_{\mathrm{pi}}^{2} /(1+\mu)$, with $\left.\mu=\omega_{\mathrm{c}}^{2} / \omega_{\mathrm{pe}}^{2}\right)$. Then, in extra-low frequency domains close to the ion gyrofrequencies where $\omega \simeq$ $\omega_{\mathrm{H}} \ll \omega_{\mathrm{c}}$ and in moderately dense plasmas where $\omega_{\mathrm{p}}^{2} / \omega_{\mathrm{c}}^{2} \sim 1$, i.e. when $\omega_{\mathrm{pe}}^{2} / \omega \omega_{\mathrm{c}} \gg 1+\omega_{\mathrm{pe}}^{2} / \omega_{\mathrm{c}}^{2}$ (as in Smith and Brice, 1964), one can estimate the cutoff frequencies in the zero order approximation using the equations $\varepsilon \pm g \simeq \varepsilon \pm g-1=0$.

Note that near the cutoff frequencies an essential decrease of the transverse plasma conductivity occurs which strongly depends on the sense of rotation of the wave electric field $\boldsymbol{E}$ around the ambient magnetic field $\boldsymbol{B}_{0}=B_{0} z$; the corresponding transverse components of the current density $\boldsymbol{j}$ verify

$j_{x} \pm i j_{y}=\frac{\omega}{4 \pi i}\left(E_{\mathrm{x}} \pm i E_{\mathrm{y}}\right)(\varepsilon \pm g-1)$.

The small term $\left\langle v_{\mathrm{b}} \omega_{\mathrm{b}}\right\rangle / \omega$ conserved in Eqs. (2)-(3), where by definition

$$
\left\langle v_{\mathrm{b}} \omega_{\mathrm{b}}\right\rangle \equiv \sum_{\mathrm{IPB}} v_{j} \omega_{j}
$$

should be essentially greater than the small correction of the term $\sim v_{\mathrm{e}} \omega / \omega_{\mathrm{c}}$ neglected in Eqs. (2)-(3); thus, in order to apply the subsequent formulas one has to verify the condition

$$
1 \ll \frac{\omega_{\mathrm{c}}\left\langle v_{\mathrm{b}} \omega_{\mathrm{b}}\right\rangle}{\omega^{2}} \frac{1}{v_{\mathrm{e}}}
$$

whereas the averaged gyrofrequency of the IPB $\left\langle\omega_{\mathrm{IPB}}\right\rangle$ is defined as

$$
\left\langle\omega_{\mathrm{IPB}}\right\rangle=\frac{\left\langle v_{\mathrm{b}} \omega_{\mathrm{b}}\right\rangle}{v_{\mathrm{IPB}}},
$$

with a resultant relative charge density $v_{\text {IPB }}$ of the IPB satisfying the charge neutrality condition: $v_{\mathrm{IPB}} \equiv v_{\mathrm{b}}=v_{\mathrm{e}}-v_{1}-$ $v_{2}$. However, neglecting the transverse displacement current in the approximation $\varepsilon \pm g \simeq \varepsilon \pm g-1$ requires also to validate the following inequality (compare with Eqs. 2-3)

$1 \ll \frac{\omega_{\mathrm{c}}\left\langle v_{\mathrm{b}} \omega_{\mathrm{b}}\right\rangle}{\omega^{2}} \frac{\omega_{\mathrm{p}}^{2}}{\omega_{\mathrm{c}}^{2}}$,

which is compatible with Eq. (7) if $1 / \nu_{\mathrm{e}} \sim \omega_{\mathrm{p}}^{2} / \omega_{\mathrm{c}}^{2} \sim 1$.

Thus, under the conditions (7)-(9), our basic equations are the following

$$
\begin{aligned}
& \varepsilon-g-1 \propto F_{\mathrm{m}}(\omega) \simeq \nu_{\mathrm{e}}-\frac{\nu_{1} \omega_{1}}{\omega+\omega_{1}}-\frac{\nu_{2} \omega_{2}}{\omega+\omega_{2}}-\frac{\left\langle\nu_{\mathrm{b}} \omega_{\mathrm{b}}\right\rangle}{\omega}=0, \\
& \varepsilon+g-1 \propto F_{\mathrm{p}}(\omega) \simeq \nu_{\mathrm{e}}+\frac{\nu_{1} \omega_{1}}{\omega-\omega_{1}}+\frac{\nu_{2} \omega_{2}}{\omega-\omega_{2}}+\frac{\left\langle\nu_{\mathrm{b}} \omega_{\mathrm{b}}\right\rangle}{\omega}=0 .
\end{aligned}
$$

In the case of a plasma with two light ions of positive charge (labelled "1" and "2"; for the negative ones we will use below the indices " 3 " and "4") and considering an IPB contributing only to the charge neutrality condition, one can use Eqs. (10)-(11) ignoring the term $\left\langle v_{\mathrm{b}} \omega_{\mathrm{b}}\right\rangle / \omega$. Noting $\omega_{ \pm}$the roots of the squared equation $\varepsilon-g-1=0$, i.e. $F_{\mathrm{m}}(\omega)=0$, one can express the relative charge densities $v_{1,2} / \nu_{\mathrm{e}}$ of the two light ions using

$$
\frac{\nu_{1,2}}{v_{\mathrm{e}}} \omega_{1,2}\left(\omega_{1,2}-\omega_{2,1}\right)=\left(\omega_{1,2}+\omega_{-}\right)\left(\omega_{1,2}+\omega_{+}\right),
$$

where the values $\left|\omega_{ \pm}\right|$correspond to the ion cutoff frequencies (see Appendix A). Note that the equation $\varepsilon+g-1=0$, i.e. $F_{\mathrm{p}}(\omega)=0$, possesses the roots $-\omega_{ \pm}$.

However, note that one can take into account the contribution of the IPB not only to the quasineutrality condition $v_{\mathrm{b}}=v_{\mathrm{e}}-v_{1}-v_{2}$, but also to the corrections to the LHR frequency and to the highest cutoff frequency values due to the nonzero term $\left\langle v_{\mathrm{b}} \omega_{\mathrm{b}}\right\rangle$ in Eqs. (10)-(11). Then the polynoms providing as solutions the cutoff frequencies, i.e. $\varepsilon \pm g-1 \simeq 0$, become of the third order in frequency $\omega$ (i.e., similar to Eq. (20) below). So, taking into account that $\left\langle v_{\mathrm{b}} \omega_{\mathrm{b}}\right\rangle / \omega \gg v_{\mathrm{e}} \omega / \omega_{\mathrm{c}}$, one can find the corrected estimates for the relative charge densities $v_{1,2} / \nu_{\mathrm{e}}$ of the two light ions

$$
\begin{aligned}
& \frac{\nu_{1,2}}{\nu_{\mathrm{e}}} \omega_{1,2}\left(\omega_{1,2}-\omega_{2,1}\right)=L \omega_{1,2}-\omega_{1} \omega_{2}+\widehat{\omega}_{+} \widehat{\omega}_{-}-(L-S)^{2} \\
& +(L-S)\left(L-\omega_{1}-\omega_{2}+\omega_{2,1} \frac{\widehat{\omega}_{+} \widehat{\omega}_{-}}{\omega_{1} \omega_{2}}\right),
\end{aligned}
$$

where $\widehat{\omega}_{+}$and $\widehat{\omega}_{-}$(actually $\left|\widehat{\omega}_{ \pm}\right|$) are the two highest ion cutoff frequencies; note that the above used notations, $\omega_{+}$ and $\omega_{-}$, correspond to the second order, and not third order, polynomial Eqs. (10)-(11); here $L$ (resp. $S$ ) is calculated using $\omega_{\mathrm{c}}, \omega_{\mathrm{pe}}^{2}$ and $\omega_{\mathrm{pi}}^{2}$ (resp. the two light ion gyrofrequencies and the two highest ion cutoff frequencies)

$L=\omega_{\mathrm{c}}\left(\omega_{\mathrm{pi}}^{2} / \omega_{\mathrm{pe}}^{2}\right)=\left[v_{1} \omega_{1}+v_{2} \omega_{2}+\left\langle v_{\mathrm{b}} \omega_{\mathrm{b}}\right\rangle\right] / \nu_{\mathrm{e}}$, 
$S=\omega_{1}+\omega_{2}+\widehat{\omega}_{+}+\widehat{\omega}_{-}$.

One can check that when $\left\langle v_{\mathrm{b}} \omega_{\mathrm{b}}\right\rangle / \omega \simeq 0$, the contribution of the IPB to $\omega_{\mathrm{pi}}$ and $\omega_{\mathrm{LH}}$ can be neglected (reminding that $\left.v_{\mathrm{e}} \omega_{\mathrm{c}} \omega_{\mathrm{pi}}^{2} / \omega_{\mathrm{pe}}^{2}=v_{1} \omega_{1}+v_{2} \omega_{2}+\left\langle v_{\mathrm{b}} \omega_{\mathrm{b}}\right\rangle\right)$, so that $L-S=0$, and Eq. (13) reduces to Eq. (12).

The value of $\left\langle v_{\mathrm{b}} \omega_{\mathrm{b}}\right\rangle$ can be estimated through the measured plasma parameters using the relation

$\frac{\left\langle v_{\mathrm{b}} \omega_{\mathrm{b}}\right\rangle}{v_{\mathrm{e}}}=\frac{\widehat{\omega}_{+} \widehat{\omega}_{-}}{\omega_{1} \omega_{2}}(L-S)$,

whereas the smallest nonvanishing cutoff frequency, $\omega_{\text {cut3 }} \equiv$ $\left|\omega_{\mathrm{D}}\right|$, due to the contribution of the IPB [or the "Dust", as expressed by the subscript "D" for the third $\operatorname{root} \omega_{\mathrm{D}}$ of Eq. (20)] to $L$ (Eq. 14), is determined as $\omega_{\text {cut } 3}=|L-S|$. The nature of the highest cutoff frequencies, i.e. the corresponding signs of $\widehat{\omega}_{ \pm}$in Eqs. (13) and (15) can be established in the approximation $\left\langle v_{\mathrm{b}} \omega_{\mathrm{b}}\right\rangle / \omega \simeq 0$ (i.e. using the approximate relations presented in Appendix A) when $\left|\widehat{\omega}_{ \pm}\right| \simeq\left|\omega_{ \pm}\right|$.

The above results have been obtained using the relations

$v_{\mathrm{e}} \widehat{\omega}_{+} \widehat{\omega}_{-} \omega_{\mathrm{D}}=\left\langle v_{\mathrm{b}} \omega_{\mathrm{b}}\right\rangle \omega_{1} \omega_{2}$,

$v_{\mathrm{e}}\left(\widehat{\omega}_{+} \widehat{\omega}_{-}+\widehat{\omega}_{+} \omega_{\mathrm{D}}+\widehat{\omega}_{-} \omega_{\mathrm{D}}\right)=\left(v_{\mathrm{e}}-v_{1}-v_{2}\right) \omega_{1} \omega_{2}$

$-\left\langle v_{\mathrm{b}} \omega_{\mathrm{b}}\right\rangle\left(\omega_{1}+\omega_{2}\right)$

$v_{\mathrm{e}}\left(\widehat{\omega}_{+}+\widehat{\omega}_{-}+\omega_{\mathrm{D}}\right)=$

$-v_{\mathrm{e}}\left(\omega_{1}+\omega_{2}\right)+v_{1} \omega_{1}+v_{2} \omega_{2}+\left\langle v_{\mathrm{b}} \omega_{\mathrm{b}}\right\rangle$,

between the three roots $\widehat{\omega}_{+}, \widehat{\omega}_{-}$and $\omega_{\mathrm{D}}$ of the polynom $F_{m}(\omega)$, i.e.

$F_{\mathrm{m}}(\omega)=v_{\mathrm{e}} \omega^{3}+\omega^{2}\left(v_{\mathrm{e}}\left(\omega_{1}+\omega_{2}\right)-v_{1} \omega_{1}-v_{2} \omega_{2}\right)$

$+\omega\left(v_{\mathrm{e}}-v_{1}-v_{2}\right) \omega_{1} \omega_{2}$

$-\left\langle v_{\mathrm{b}} \omega_{\mathrm{b}}\right\rangle\left(\omega^{2}+\omega\left(\omega_{1}+\omega_{2}\right)+\omega_{1} \omega_{2}\right)=0$,

as well as the expression of $\omega_{\mathrm{pi}}^{2} / \omega_{\mathrm{pe}}^{2}$ as a function of $v_{j}$ and $\omega_{j}$

$\frac{\omega_{\mathrm{pi}}^{2}}{\omega_{\mathrm{pe}}^{2}}=\frac{\nu_{1} \omega_{1}+\nu_{2} \omega_{2}+\left\langle\nu_{\mathrm{b}} \omega_{\mathrm{b}}\right\rangle}{\nu_{\mathrm{e}} \omega_{\mathrm{c}}}$.

Note that the roots of the equation $F_{\mathrm{p}}(\omega)=0$ have signs opposite to those of $F_{\mathrm{m}}(\omega)=0$.

Since experimental data can not be provided for $\omega_{\mathrm{D}}$ and $\left\langle\nu_{\mathrm{b}} \omega_{\mathrm{b}}\right\rangle$ by typical diagnostic tools above the extra-low frequency domain, these parameters should be excluded from Eqs. (17)-(19): from Eq. (17) one has $\left\langle v_{\mathrm{b}} \omega_{\mathrm{b}}\right\rangle=$ $v_{\mathrm{e}}\left(\widehat{\omega}_{+} \widehat{\omega} / \omega_{1} \omega_{2}\right) \omega_{\mathrm{D}}$; then, Eq. (19) permits to find, using Eq. (14), that $\omega_{\mathrm{D}}=L-S$, so that finally Eq. (13) can be obtained from Eqs. (17)-(18).

Finally we can express $v_{1} / \nu_{\mathrm{e}}, \nu_{2} / \nu_{\mathrm{e}}$ and $\left\langle v_{\mathrm{b}} \omega_{\mathrm{b}}\right\rangle / \nu_{\mathrm{e}}$ through the characteristic plasma frequencies which can be measured by diagnostic tools. Moreover, using the quasineutrality condition one can estimate the relative charge density $\nu_{\mathrm{b}}$ of the IPB as well as its averaged gyrofrequency $\left\langle\omega_{\mathrm{IPB}}\right\rangle$ as

$$
\begin{aligned}
& \frac{\nu_{\mathrm{b}}}{\nu_{\mathrm{e}}}=1-\frac{\nu_{1}}{\nu_{\mathrm{e}}}-\frac{\nu_{2}}{\nu_{\mathrm{e}}}=\frac{\widehat{\omega}_{+} \widehat{\omega}_{-}}{\omega_{1} \omega_{2}} \\
& +\frac{\left(\omega_{1}+\omega_{2}\right)}{\omega_{1} \omega_{2}}(L-S) \frac{\widehat{\omega}_{+} \widehat{\omega}_{-}}{\omega_{1} \omega_{2}}+\frac{\left(\widehat{\omega}_{+}+\widehat{\omega}_{-}\right)}{\omega_{1} \omega_{2}}(L-S), \\
& \left|\left\langle\omega_{\mathrm{IPB}}\right\rangle\right|=\frac{\left\langle\nu_{\mathrm{b}} \omega_{\mathrm{b}}\right\rangle}{\left|\nu_{\mathrm{b}}\right|}=\left|\frac{\nu_{\mathrm{e}}}{\nu_{\mathrm{b}}} \frac{\widehat{\omega}_{+} \widehat{\omega}}{\omega_{1} \omega_{2}}(L-S)\right| .
\end{aligned}
$$

Note that it is relevant to apply Eq. (20) only if $\left|\left\langle\omega_{\mathrm{IPB}}\right\rangle\right|$ is not extremely small, i.e. if the conditions (7)-(9) are fulfilled, what can be checked using Eq. (16) by

$$
1 \ll \frac{\omega_{\mathrm{c}}}{\omega^{2}}\left|\frac{\widehat{\omega}_{+} \widehat{\omega}_{-}}{\omega_{1} \omega_{2}}(L-S)\right|, 1 \ll \frac{\omega_{\mathrm{c}}}{\omega^{2}}\left|\frac{\widehat{\omega}_{+} \widehat{\omega}_{-}}{\omega_{1} \omega_{2}}(L-S)\right| v_{\mathrm{e}} \frac{\omega_{\mathrm{p}}^{2}}{\omega_{\mathrm{c}}^{2}} .
$$

Using the approximate relation $\left|v_{\mathrm{e}} \widehat{\omega}_{+} \widehat{\omega}_{-}\right| \simeq\left|v_{\mathrm{e}} \omega_{+} \omega_{-}\right|=$ $\left|\nu_{\mathrm{b}} \omega_{1} \omega_{2}\right|$, the first inequality can be written as $\left(\omega_{\text {cut3 } 3}=\right.$ $|L-S|)$

$$
\begin{aligned}
& 1 \ll \frac{\omega_{c} \omega_{\text {cut } 3}}{\omega^{2}}\left|\frac{\nu_{\mathrm{b}}}{v_{\mathrm{e}}}\right| \sim \frac{\omega_{\mathrm{c}} \omega_{\mathrm{H}}}{\omega^{2}}\left|\frac{\nu_{\mathrm{b}}}{v_{\mathrm{e}}}\right|\left\langle Z_{\mathrm{IPB}} M_{\mathrm{H}} / M_{\mathrm{IPB}}\right\rangle \\
& \sim \frac{\omega_{\mathrm{c}}\left|\left\langle\omega_{\mathrm{IPB}}\right\rangle\right|}{\omega_{\mathrm{H}}^{2}}\left|\frac{\nu_{\mathrm{b}}}{v_{\mathrm{e}}}\right|,
\end{aligned}
$$

where, as it is written in Eq. (D6) of Appendix D,

$$
\left\langle Z_{\mathrm{IPB}} M / M_{\mathrm{IPB}}\right\rangle=\left|\left\langle\omega_{\mathrm{IPB}}\right\rangle\right| / \omega_{\mathrm{H}}
$$

is the averaged charge-to-mass ratio of the IPB (in electron charge and atomic mass units); here, except of extraordinary cases mentioned below, the following parameters can be considered as being of moderate values, namely

$\frac{\omega_{\mathrm{H}}^{2}}{\omega^{2}} \sim \frac{\omega_{\text {cut3 }}}{\left|\left\langle\omega_{\mathrm{IPB}}\right\rangle\right|} \sim 1$.

Then the developed approach only fails to estimate ultra small values of $\left|\left\langle\omega_{\mathrm{IPB}}\right\rangle\right|$; actually, our results are not reliable when

$\frac{\omega_{\text {cut } 3 \omega_{\mathrm{c}}}}{\omega^{2}} \frac{\left|\nu_{\mathrm{b}}\right|}{v_{\mathrm{e}}} \sim \frac{\left|\left\langle\omega_{\text {IPB }}\right\rangle\right| \omega_{\mathrm{c}}}{\omega_{\mathrm{H}}^{2}} \frac{\left|\nu_{\mathrm{b}}\right|}{\nu_{\mathrm{e}}} \lesssim 1$,

where $\omega_{\mathrm{H}}$ represents the gyrofrequency of the actual lightest ion $\left(\right.$ here $\mathrm{H}^{+}$); instead of the third ion cutoff frequency we use as an estimate the value of $\left|\left\langle\omega_{\text {IPB }}\right\rangle\right|$.

Note that to apply the above relations one should know what are the two light ion species or, if not available, search the appropriate set of light ions suitable to the equations. At the same time the above agreement implies to use the indices " 1 " and " 2 " to denote the positive ions, but " 3 " and "4" for the negative ones; the substitution of ion " 3 " instead of " 2 ", for instance, needs to substitute in all the formulas $-v_{3}$ and 


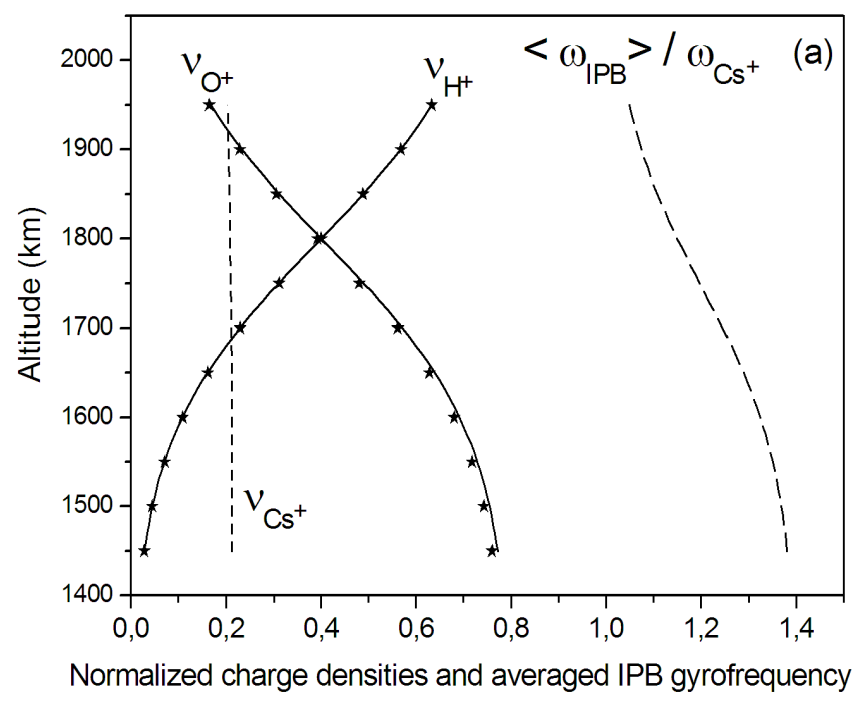

Fig. 1a. Altitude profiles of the relative charge densities of the three ion species $\mathrm{H}^{+}, \mathrm{O}^{+}$and $\mathrm{Cs}^{+}$, as well as of the normalized averaged gyrofrequency $\left\langle\omega_{\mathrm{IPB}}\right\rangle / \omega_{\mathrm{Cs}^{+}}$. The IPB is formed by $\mathrm{Cs}^{+}$ions which contaminate the ionospheric background plasma constituted by the two light ions $\mathrm{H}^{+}$and $\mathrm{O}^{+}$distributed in altitude according to a diffusive equilibrium type model. The solid lines show the corresponding values of $v_{\mathrm{O}^{+}}$and $v_{\mathrm{H}^{+}}$, taking into account that the assumed contamination by the $\mathrm{Cs}^{+}$ions does not depend on the altitude, whereas the superposed stars correspond to calculations based on Eq. (13). The dotted (resp. dashed) lines represent the recovered relative charge density $v_{\mathrm{Cs}^{+}}$and normalized averaged gyrofrequency of the IPB, $\left\langle\omega_{\mathrm{IPB}}\right\rangle / \omega_{\mathrm{Cs}^{+}}$, respectively; (a) and (b) correspond to different levels of plasma contamination: (a) $v_{\mathrm{Cs}^{+}}=0.2$; (b) $v_{\mathrm{Cs}^{+}}=0.5$.

$-\omega_{3}$ instead of $\nu_{2}$ and $\omega_{2}$. Then one can use the normalization condition as mentioned just above: in the case when Eq. (22) provides a positive value for $v_{\mathrm{b}}$, then $v_{\mathrm{e}}=1$, because $v_{\mathrm{e}}=v_{\mathrm{b}}+v_{1}+v_{2}=1$; otherwise, $v_{\mathrm{e}}+\left|v_{\mathrm{b}}\right|=v_{1}+v_{2}=1$ and $v_{\mathrm{e}}=\left(v_{1} / \nu_{\mathrm{e}}+v_{2} / \nu_{\mathrm{e}}\right)^{-1}$, so that $\nu_{\mathrm{b}}=v_{\mathrm{e}}-1<0$.

The measured highest cutoff frequencies are $\omega_{\text {cut1 }}$ and

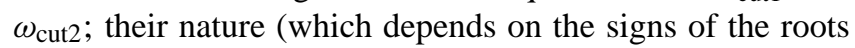
$\widehat{\omega}_{+}$and $\widehat{\omega}_{-}$) can be draftily selected according to the Table 1 of Appendix A, identifying $\widehat{\omega}_{ \pm}$and $\omega_{ \pm}$. One should take care that, when constituting such a table, the use of the approximation $\widehat{\omega}_{ \pm} \simeq \omega_{ \pm}$can induce contradictions; but those can easily be revised.

One of the difficulties related to the developed approach is to measure the characteristic plasma frequencies with a rather high accuracy, especially within the two distant high frequency domains near $\omega_{\mathrm{c}}$ and $\omega_{\mathrm{pe}}$ as well as in the middle frequency range near $\omega_{\text {pi }}$ and $\omega_{\mathrm{LH}}$. Only in this case the resulting inaccuracy of $L=\omega_{c} \omega_{\mathrm{pi}}^{2} / \omega_{\mathrm{pe}}^{2}$ will be small in comparison with the two highest ion gyrofrequencies and the highest ion cutoff frequencies contributing to $L-S$

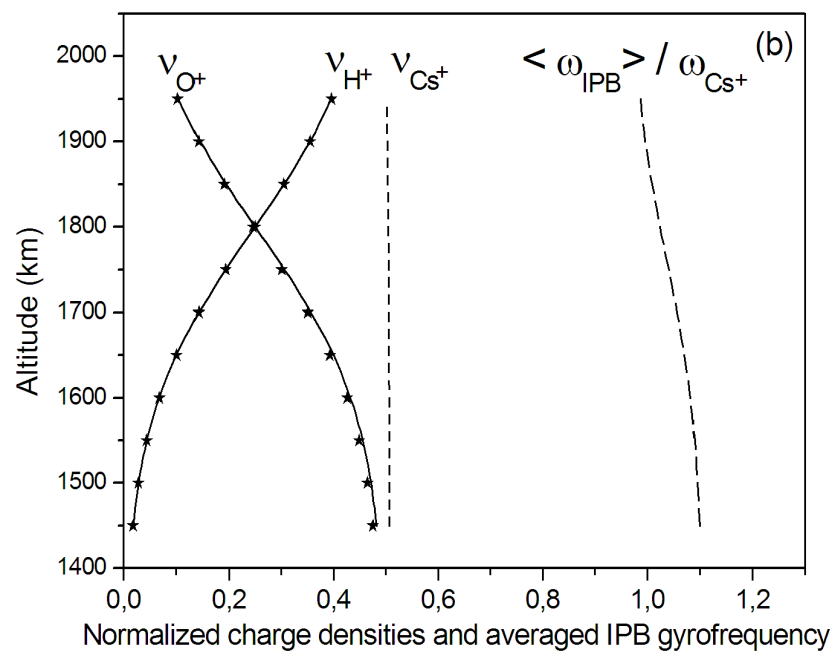

Fig. 1b. $v_{\mathrm{Cs}^{+}}=0.5$.

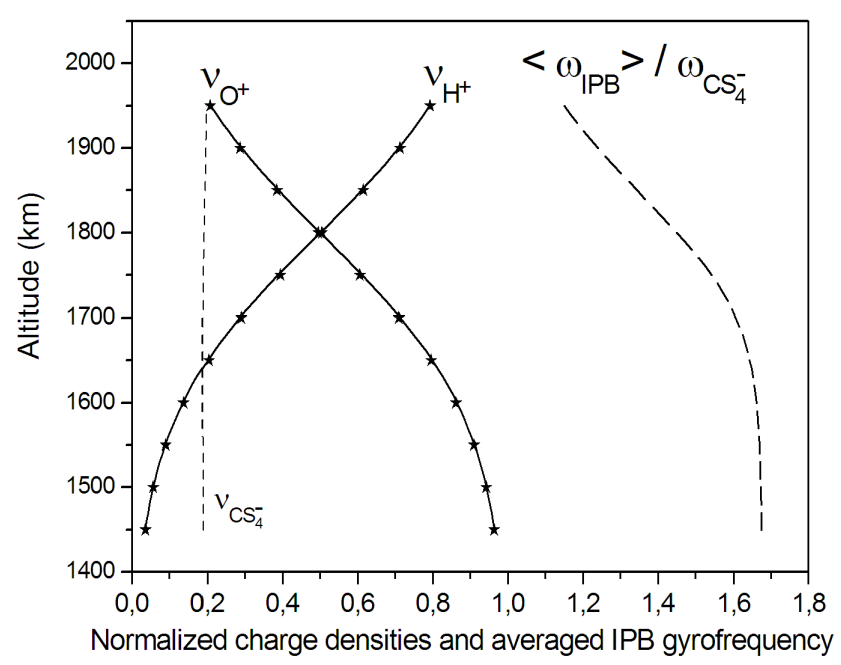

Fig. 2. Curves similar to those of Fig. 1, but for a plasma contamination by negative $\mathrm{CS}_{4}^{-}$ions of relative charge density $v_{\mathrm{CS}_{4}^{-}}=0.2$.

$$
\begin{aligned}
& L-S=\omega_{\mathrm{c}} \frac{\omega_{\mathrm{pi}}^{2}}{\omega_{\mathrm{pe}}^{2}}-\left(\omega_{1}+\omega_{2}+\widehat{\omega}_{+}+\widehat{\omega}_{-}\right) \\
& =\omega_{1}\left(\frac{\nu_{1}}{v_{\mathrm{e}}}-1\right)+\omega_{2}\left(\frac{\nu_{2}}{v_{\mathrm{e}}}-1\right)-\widehat{\omega}_{+}-\widehat{\omega}_{-}+\frac{\left\langle\nu_{\mathrm{b}} \omega_{\mathrm{b}}\right\rangle}{\nu_{\mathrm{e}}},
\end{aligned}
$$

thus providing resultant estimates of the relative content of the two light ions with a reliable accuracy.

\section{Estimating ionospheric plasma contamination}

Let us apply the developed technique to analyze the contamination of ionospheric Earth plasmas by heavy charged particles. In order to test our model by recovering reliably 
the relative charge densities of the ion species, let us consider a typical ionospheric plasma composition dominated by the ions $\mathrm{H}^{+}$and $\mathrm{O}^{+}$, for definiteness at altitudes corresponding to the upper ionosphere in the transition region from the Oxygen to the Hydrogen ions' prevalence, and contaminated mostly by one variety of heavy ions or charged particulates.

If experimental data are available, high frequency wave measurements should provide the electron gyro- and plasma frequencies, the LHR frequency and the two highest ion cutoff frequencies $\omega_{\text {cut } 1}$ and $\omega_{\text {cut } 2 \text {, which are determined as }}$ the low frequency boundaries of the frequency-time spectra of the electron whistlers and the adjacent ion cyclotron whistlers. In order to demonstrate the reliability and the effectiveness of our method, let us calculate these characteristic frequencies using the background diffusive equilibrium plasma model with two ions (Angerami and Thomas, 1964) applicable to the near Earth plasmas. For definiteness the relative charge density of the contaminating heavy charged particles is chosen to be constant in the considered altitude domain. Note that the contamination by heavy positively charged ions is accompanied here by the increase of the electron density, the light ions' densities being fixed; in opposite, the contamination by electronegative gas molecules providing negatively charged ions is accompanied by a diminution of the electron density.

Using the scheme described in the previous section, one can recover the altitude profiles of the relative charge densities of the lightest ions as well as of the heavy particulates, whose averaged gyrofrequency is also evaluated, as shown by the figures presented below. In order to test the applicability of our method to near Earth plasmas, we select a set of heavy ions of charge-to-mass ratios significantly larger than those of the lightest ions, $\mathrm{H}^{+}$and $\mathrm{O}^{-}$(we have chosen below as samples the ions $\mathrm{Cs}^{+}\left(M_{\mathrm{A}}=133\right)$ and $\mathrm{CS}_{4}^{-}\left(M_{\mathrm{A}}=140\right)$, but it could be any other set of heavy charged species).

Figure 1 shows the altitude profiles of the relative charge densities $v_{\mathrm{O}^{+}}, v_{\mathrm{H}^{+}}$and $v_{\mathrm{Cs}^{+}}$of the two dominant ions, $\mathrm{O}^{+}$ and $\mathrm{H}^{+}$, and the contaminating heavy ions $\mathrm{Cs}^{+}$which play the role of the IPB. The solid lines correspond to the values of $v_{\mathrm{O}^{+}}$and $v_{\mathrm{H}^{+}}$provided by the diffusive equilibrium model with the two ions $\mathrm{H}^{+}$and $\mathrm{O}^{+}$; however, the contaminating $\mathrm{Cs}^{+}$ions, whose relative charge density $v_{\mathrm{Cs}^{+}}$is independent of the altitude, modify the normalized charge neutrality equation, so that $v_{\mathrm{e}}=v_{\mathrm{O}^{+}}+v_{\mathrm{H}^{+}}+v_{\mathrm{Cs}^{+}}=1$ (the altitude of the $\mathrm{H}^{+}$and $\mathrm{O}^{+}$equipartition is $1800 \mathrm{~km}$, as in Lundin and Krafft, 2009b). The stars correspond to calculations based on Eq. (13) and fit well with the solid lines. The relative density $v_{\mathrm{Cs}^{+}}$of the heavy ions is reliably recovered and represented by a short dashed curve. The long dashed line corresponds to the estimated effective gyrofrequency of the IPB normalized to the gyrofrequency of the heavy ion $\mathrm{Cs}^{+},\left\langle\omega_{\mathrm{IPB}}\right\rangle / \omega_{\mathrm{Cs}^{+}}$. In the case of Fig. 1a we have $v_{\mathrm{Cs}^{+}}=0.2$ whereas Fig. 1b corresponds to more intense ion contamination with $v_{\mathrm{Cs}^{+}}=0.5$.

Figure 2 shows the same curves as in Fig. 1, but for the case when the contaminating IPB is formed by $\mathrm{CS}_{4}^{-}$ions with $M_{\mathrm{A}}=140$ and $v_{\mathrm{CS}_{4}^{-}}=0.2$. One of the visible difference between Fig. 1a and Fig. 2 is connected with the definition of $v_{j}$, the charge density being normalized to the total positive ion charge density. Then, when the contamination is due to negative heavy ions as in Fig. 2, the charge neutrality condition provides that $v_{\mathrm{O}^{+}}+v_{\mathrm{H}^{+}}=1$; however, in Fig. 1a, $v_{\mathrm{O}^{+}}+v_{\mathrm{H}^{+}}$is less than unity by the value of the relative charge density $v_{\mathrm{Cs}^{+}}=0.2$.

\section{Conclusion}

As conclusion, we demonstrated how to determine the ion composition of a magnetized dusty plasma and to estimate the main characteristic parameters of its most heavy part formed by charged particulates of large atomic masses, without using mass spectrometer tools but only wave measurements providing the two highest ion cutoff frequencies, the LHR frequency and the electron characteristic frequencies of the plasma.

The presented approach is valid if $\omega_{\mathrm{pe}}^{2} / \omega_{\mathrm{H}} \omega_{\mathrm{c}} \simeq$ $2000 \omega_{\mathrm{pe}}^{2} / \omega_{\mathrm{c}}^{2} \gg 1$ (compare with Smith and Brice, 1964) and under the conditions defined in Eqs. (7), (9) and (24). The values of the relative charge densities of the light ion species and of the heavy charged constituents as a whole can be recovered, as well as the averaged charge-to-mass ratio of the heavy plasma background. Even in the case when the above mentioned conditions are not satisfied with excess, the possibility to estimate the averaged charge-to-mass ratio of a heavy plasma background using whistler wave measurements is useful when mass spectrometer tools are not available on board the space vehicles.

Moreover, in a near Earth plasma surrounding with finite $\mu=\omega_{\mathrm{c}}^{2} / \omega_{\mathrm{pe}}^{2}$, the recovering of the above mentioned ion composition data is sufficient to determine a quantitatively reliable local dispersion equation for the electron whistler waves in their total unbroken frequency domain (see Lundin and Krafft, 2009a, b, for $\left.\omega \ll \omega_{\text {pe }}\right)$. Thus, in application to the problem of ion composition modelling in the near Earth plasmas, we can justify that any modification of the selected ion composition model (i.e. ion species' distributions with altitude) will not influence on the electron whistlers' propagation if the altitude profiles of the electron plasma frequency, the LHR and the two highest ion cutoff frequencies are not essentially modified.

\section{Appendix A}

\section{Ion gyro- and cutoff frequencies disposition}

Let us briefly summarize the results of the analysis performed by Lundin and Krafft (2009a), where the mutual disposition of the ion gyro- and cutoff frequencies was determined in the case when the IPB contributes only to the charge 
Table A1. Disposition of the ion cutoff frequencies $\omega_{\text {cut } 2}$ and $\omega_{\text {cut } 1}$ with respect to the gyrofrequencies $\omega_{1}$ and $\omega_{2}$ (resp. $\omega_{3}$ and $\omega_{4}$ ) of the positively (resp. negatively) charged light ions. In the last column the relative charge density $\nu_{\mathrm{b}}$ of the IPB is compared with

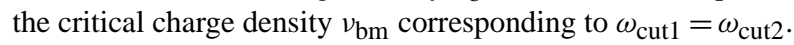

\begin{tabular}{|c|c|c|}
\hline valid pairs & $\left\{\omega_{1}, \omega_{2}, \omega_{3}, \omega_{4}, \omega_{\text {cut } 2}<\omega_{\text {cut } 1}\right\}$ & $v_{\mathrm{b}}$ \\
\hline$\omega_{\text {cut } 2}^{-}, \omega_{\text {cut } 1}^{-}$ & $\omega_{4}<\omega_{\text {cut } 2}^{-}<\omega_{3}<\omega_{\text {cut } 1}^{-}$ & $0<v_{\mathrm{b}}$ \\
\hline$\omega_{\text {cut } 2}^{+}, \omega_{\text {cut } 1}^{-}$ & $\omega_{2}<\omega_{\text {cut } 2}^{+}<\omega_{1}$ & $\nu_{\mathrm{b}}<v_{\mathrm{bm}}<0$ \\
\hline$\omega_{\text {cut } 2}^{-}, \omega_{\text {cut } 1}^{+}$ & $\omega_{2}<\omega_{\text {cut } 1}^{+}<\omega_{1}$ & $v_{\mathrm{bm}}<v_{\mathrm{b}}<0$ \\
\hline$\omega_{\text {cut } 2}^{+}, \omega_{\text {cut } 1}^{+}$ & $\omega_{\text {cut } 2}^{+}<\omega_{2}<\omega_{\text {cut } 1}^{+}<\omega_{1}$ & $0<v_{\mathrm{b}}$ \\
\hline$\omega_{\text {cut } 2}^{+}, \omega_{\text {cut } 1}^{+}$ & - & $\nu_{\mathrm{b}}<\nu_{\mathrm{bm}}<0$ \\
\hline$\omega_{\text {cut } 2}^{-}, \omega_{\text {cut } 1}^{-}$ & $\omega_{2}<\omega_{3}, \omega_{\text {cut } 2}^{-}<\omega_{3}<\omega_{\text {cut } 1}^{-}$ & $v_{\mathrm{bm}}<v_{\mathrm{b}}<0$ \\
\hline$\omega_{\text {cut } 2}^{+}, \omega_{\text {cut } 1}^{-}$ & $\omega_{\text {cut } 2}^{+}<\omega_{2}<\omega_{3}<\omega_{\text {cut } 1}^{-}$ & $0<v_{\mathrm{b}}$ \\
\hline$\omega_{\text {cut } 2}^{-}, \omega_{\text {cut } 1}^{+}$ & $\omega_{3}<\omega_{2}, \omega_{3}<\omega_{\text {cut } 2}^{-}, \omega_{\text {cut } 1}^{+}<\omega_{2}$ & $0<v_{\mathrm{bm}}<v_{\mathrm{b}}$ \\
\hline$\omega_{\text {cut } 2}^{+}, \omega_{\text {cut } 1}^{-}$ & $\omega_{3}<\omega_{2}, \omega_{3}<\omega_{\text {cut } 1}^{-}, \omega_{\text {cut } 2}^{+}<\omega_{2}$ & $0<v_{\mathrm{b}}<v_{\mathrm{bm}}$ \\
\hline$\omega_{\text {cut } 2}^{-}, \omega_{\text {cut } 1}^{-}$ & $\omega_{3}<\omega_{2}, \omega_{\text {cut } 2}^{-}<\omega_{3}<\omega_{\text {cut } 1}^{-}$ & $\nu_{\mathrm{b}}<0$ \\
\hline
\end{tabular}

neutrality condition, so that the equation $F(\omega)=0$ can be reduced to the next ones

$\varepsilon \pm g \simeq \varepsilon \pm g-1 \propto$

$v_{\mathrm{e}} \omega^{2} \mp \omega\left(\omega_{1}+\omega_{2}\right)\left(v_{\mathrm{b}}-v_{\mathrm{bm}}\right)+v_{\mathrm{b}} \omega_{1} \omega_{2}=0$,

where $v_{\mathrm{b}}=v_{\mathrm{e}}-v_{1}-v_{2}$ is the resultant relative charge density of the IPB and $v_{\mathrm{bm}}=-\left(v_{1} \omega_{2}+v_{2} \omega_{1}\right) /\left(\omega_{1}+\omega_{2}\right)$. Using the notations $\omega_{ \pm}$for the roots of $\varepsilon-g-1=0$, one can express the relative densities of the two light ions as in Eq. (12)

$v_{1,2}=v_{\mathrm{e}} \frac{\left(\omega_{1,2}+\omega_{-}\right)\left(\omega_{1,2}+\omega_{+}\right)}{\omega_{1,2}\left(\omega_{1,2}-\omega_{2,1}\right)}$.

Note that the equation $\varepsilon+g-1=0$ possesses the roots $-\omega_{ \pm}$. However, only the moduli of $\omega_{ \pm}$coincide with the cutoff frequency values. Note also that, if one of the two light ions, for example that with subscript " 1 ", is negative (so that we decide to replace its subscript by "3"), one should replace in Eq. (A1) and anywhere else $\omega_{1}$ by $-\omega_{3}$ and $\nu_{1}$ by $-v_{3}$, respectively.

The pair of equations $\varepsilon \pm g \simeq \varepsilon \pm g-1=0$ defines two real positive cutoff frequencies independently of the charge sign of the IPB and of the light ions. However, the nature of the cutoff frequencies (i.e. what equation, $\varepsilon-g=0$ or $\varepsilon+g=0$, provides the positive root - or both of them) and their relative position with respect to the gyrofrequencies of the light ions can be different depending on the signs of the coefficients of both quadratic Eqs. (A1), which differ one from the other in the sign of the term proportional to $\omega$ only.

The nature of the two positive roots of Eq. (A1) and their positions on the real axis can be easily determined depending on the signs of the polynomial coefficients $\left(\omega_{1}+\omega_{2}\right)\left(v_{\mathrm{b}}-\right.$ $\left.\nu_{\mathrm{bm}}\right)$ and $\omega_{1} \omega_{2} \nu_{\mathrm{b}}$. One should take also into account that the relative charge densities of the light ions $v_{1,2}$ (or $v_{3,4}$ ) in
Eq. (A2) are positive by definition. The most general result of this analysis is that the lower cutoff frequency $\omega_{\text {cut2 }}$ can not be greater than the highest gyrofrequency of the light ions; at the same time, the upper cutoff frequency $\omega_{\text {cut } 1}\left(\omega_{\text {cut } 2}<\right.$ $\left.\omega_{\text {cut } 1}\right)$ can not be smaller than the lowest gyrofrequency of the light ions.

Table A1 summarizes all the possible dispositions of the ion cutoff frequencies $\omega_{\text {cut } 2}$ and $\omega_{\text {cut } 1}$ with respect to the gyrofrequencies of the positively and negatively charged light ions.

Note the superscripts "+" and "-" used in Table A1: the frequency $\omega_{\text {cut }}^{-}$(resp. $\left.\omega_{\text {cut }}^{+}\right)$is the positive root of $\varepsilon-g \simeq 0$ (resp. $\varepsilon+g \simeq 0$ ). The two real positive roots of the equations $\varepsilon \pm g \simeq \varepsilon \pm g-1=0$ are labelled by the subscripts " 1 " and

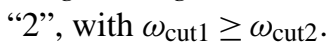

In all the above expressions the terms $\omega_{+}$or $\omega_{-}$should be substituted by $\omega_{\text {cut } 1}^{-}$or $\omega_{\text {cut } 2}^{-}$(with superscript “-”) if this possibility is permitted according to the first column of the table. However, if $\omega_{\text {cut } 1,2}^{+}$appears in this column (with superscript " + "), the substitution of $-\omega_{\text {cut }}^{+}$instead of $\omega_{+}$or $\omega_{-}$should be done.

The second column shows the mutual disposition of the ion gyro- and cutoff frequencies. The parameter $v_{\mathrm{bm}}$, introduced in the last column, corresponds to the relative density of the IPB when both cutoff frequencies merge, i.e. $\omega_{\text {cut } 1}=\omega_{\text {cut } 2}$.

\section{Appendix B}

\section{Cold plasma wave dispersion equation near the ion cutoff frequencies}

As it was shown in previous works by Lundin and Krafft (2008, 2009a, b), the cold plasma dispersion law without any simplifications can be presented in a form where the term $F(\omega)$ vanishing at the ion cutoff frequencies visibly appears

$K\left[F\left(K+\sin ^{2} \theta\right)+\varepsilon\left(1+\cos ^{2} \theta\right)\right]+\varepsilon-(\varepsilon-\eta) \cos ^{2} \theta=0,(\mathrm{~B} 1)$

with

$F \equiv F(\omega)=\left(\varepsilon^{2}-g^{2}\right) / \eta, \quad K \equiv K(\omega)=-\eta / N^{2}$.

$\theta$ is the angle between the wavevector $\boldsymbol{k}$ and the ambient magnetic field $\boldsymbol{B}_{0}=B_{0} z ; z$ is a unit vector; we use the conventional notations of Shafranov (1967) for the plasma permittivity tensor components $\varepsilon, g$ and $\eta$; the so-called cutoff points of the refractive index $N$, where $N=0$ (or $K \rightarrow \infty$ ), can be reached only at the cutoff frequencies where $F(\omega) \propto$ $\varepsilon^{2}-g^{2}=0$. The displacement current component parallel to $\boldsymbol{B}_{0}$ can be omitted for the frequency band sufficiently lower than the electron plasma frequency $\omega_{\mathrm{pe}}, \omega \ll \omega_{\mathrm{pe}}$; then we get

$K \equiv-\frac{\eta}{N^{2}} \simeq \frac{\omega_{\mathrm{pe}}^{2}}{k^{2} c^{2}} \equiv \frac{\omega_{\mathrm{p}}^{2}}{k^{2} c^{2}} \frac{n_{\mathrm{e}}}{n} \equiv \kappa$, 
where $\omega_{\mathrm{p}}$ is the value of the electron plasma frequency calculated for an electron density $n_{\mathrm{e}}$ equal to the total density $n$ of the positive charges.

The charge neutrality condition can be written as

$n \equiv \sum_{\alpha} Z_{\alpha} n_{\alpha}=n_{\mathrm{e}}+\sum_{\beta} Z_{\beta} n_{\beta}$.

Here and below we use the index $\alpha$ (resp. $\beta$ ) to numerate the positive ions (resp. the negative ions or other heavy negatively charged particles as dust grains); $n_{j}$ and $Z_{j}>0$ are the density and the charge number of the ion species $j$. Actually an additional and not very restrictive condition, $\omega_{\mathrm{pe}}^{2} \gg \omega_{\mathrm{pi}}^{2}$, i.e. $n_{\mathrm{e}} / n \gg(m / M)\left\langle Z_{j} M / M_{j}\right\rangle$, has been applied in Eq. (B3), with

$\left\langle\frac{Z_{j} M}{M_{j}}\right\rangle \equiv \sum_{j=\alpha, \beta} \frac{Z_{j} n_{j}}{\left(2 n-n_{\mathrm{e}}\right)} \frac{Z_{j} M}{M_{j}}, \sum_{j=\alpha, \beta} \frac{Z_{j} n_{j}}{2 n-n_{\mathrm{e}}}=1$,

whereas the ion plasma frequency is

$\omega_{\mathrm{pi}}^{2} \equiv \sum_{j} \omega_{\mathrm{p} j}^{2}=$

$\sum_{j} \frac{4 \pi Z_{j}^{2} n_{j} e^{2}}{M_{j}}=\frac{4 \pi n e^{2}}{M}\left(2-\frac{n_{\mathrm{e}}}{n}\right)\left\langle\frac{Z_{j} M}{M_{j}}\right\rangle$,

where $M$ (resp. $m$ ) is the proton (resp. electron) mass; the summation over $j$ includes both positive and negative particles of mass $M_{j}$.

Note that $\omega_{\mathrm{c}}^{2} / \omega_{\mathrm{p}}^{2}$ is considered here to be of finite value, as in the near Earth plasma the parameter $\mu=\omega_{\mathrm{c}}^{2} / \omega_{\mathrm{pe}}^{2}$ has been found not to be small at altitudes lower than $4000 \mathrm{~km}$ (Sonnwalkar, 2004); so, our study is not limited to the usually considered overdense plasma conditions for which $\mu \ll 1$.

The normalized wavelengths $\kappa=\omega_{\mathrm{pe}}^{2} / k^{2} c^{2}$ corresponding to the electron whistler waves of extra-low frequencies close to $\omega_{\mathrm{H}}$ (for oblique wave propagation with respect to $\boldsymbol{B}_{0}$ ) should have rather large $\kappa, \kappa \simeq M / m \gg 1$ (i.e. $\omega_{\mathrm{pH}}^{2} / k^{2} c^{2} \simeq$ 1 , where $\omega_{\mathrm{pH}}$ is the proton plasma frequency); however, to span the frequency domain around or above the LHR frequency one should have $\kappa<\sqrt{M / m} ; \kappa \sim 1$ near the characteristic electron gyro- and plasma frequencies, whereas $\kappa \ll 1$ in the corresponding quasi-resonant limit.

\section{Appendix C}

\section{On the variation of the magnetic field polarization ellipse with frequency}

To obtain reliable estimates of the ion composition parameters using measurements of the characteristic plasma frequencies registered in distant ranges as the LHR frequency $\left(\omega_{\mathrm{LH}}\right)$ and the ion cutoff frequencies' $\left(\omega_{\text {cut }}\right)$ domains, one should take care to determine with the highest possible relative accuracy at least the value of $\omega_{\mathrm{LH}} \gg \omega_{\text {cut }}$. The spectral lines of the large electric field intensity $|E|^{2}$ visible on the electron whistler spectrograms are located near the frequency $\omega$ where $\varepsilon(\omega)=0$ locally. This fact can be justified by the focusing law (Lundin and Krafft, 1997) governing the intensity of wave packets propagating in the deep quasi-resonant regime, i.e. with $\kappa=\omega_{\mathrm{pe}}^{2} / k^{2} c^{2} \ll 1$

$|E|^{2} \sqrt{|\varepsilon \eta|} h_{1 R}^{2} h_{2} \simeq$ const,

where $h_{1 R}$ and $h_{2}$ are the Lamé coefficients describing the convergence in space of quasi-resonant ray trajectories of fixed frequency; in the case of the dipole Earth magnetic field ( $\lambda$ is the magnetic latitude) and for the low frequency limit $\omega \ll \omega_{\mathrm{c}}$, the intensity of the wave packets propagating almost along the geomagnetic field lines verify

$|E|^{2} \propto \frac{1+3 \sin ^{2} \lambda}{\cos ^{9} \lambda} \frac{\omega^{2}}{\sqrt{\omega_{\mathrm{pe}}^{2}\left(1+\omega_{\mathrm{pe}}^{2} / \omega_{\mathrm{c}}^{2}\right)} \sqrt{\omega^{2}-\omega_{\mathrm{LH}}^{2}}}$.

However, when the waves are scattered from the deep quasiresonant regime, the registered maximum field intensity $|E|^{2}$ can be shifted from the local LHR frequency $\omega_{\mathrm{LH}}$ of the background plasma, and this will not permit to measure the value of $\omega_{\mathrm{LH}}$ with the required accuracy.

However, another technique can be used to determine the local ion plasma frequency $\omega_{\text {pi }}$. It is based on the polarization properties of the plane wave's field components in the plane orthogonal to its wavevector $\boldsymbol{k}$, i.e. on the registration of the wave's magnetic field components. Using the notations $\alpha_{x}=$ $-i E_{\mathrm{x}} / E_{\mathrm{y}}$ and Eq. (1.31) of Shafranov (1967), where the zaxis is directed along $\boldsymbol{k}$ and the ambient magnetic field is given by $\boldsymbol{B}_{0}=\left(B_{\mathrm{x}}, 0, B_{\mathrm{Z}}\right)$, with $\cos \theta=B_{\mathrm{Z}} / B_{0}$, one has

$$
\begin{aligned}
& \frac{\alpha_{x}^{2}-1}{\alpha_{x} \cos \theta}=-\chi \frac{\varepsilon}{g} \frac{\sin ^{2} \theta}{\eta \cos ^{2} \theta_{\mathrm{R}} \cos ^{2} \theta}= \\
& -\frac{g}{(\varepsilon-\chi)} \chi \frac{\sin ^{2} \theta}{\eta \cos ^{2} \theta}=-\frac{\omega \omega_{\mathrm{c}}}{\omega_{\mathrm{pe}}^{2}} \chi \frac{\sin ^{2} \theta}{\cos ^{2} \theta},
\end{aligned}
$$

where we used the parameters contributing to the modified electron whistler dispersion law in a plasma of moderate density (Lundin and Krafft, 2002), namely $\eta, \chi=\varepsilon-g^{2} /(\varepsilon-\eta)$ and $\cos ^{2} \theta_{\mathrm{R}}=\varepsilon /(\varepsilon-\eta)$, as well as the following relation

$$
\frac{g}{\varepsilon}=\frac{(\varepsilon-\chi)}{g} \frac{1}{\cos ^{2} \theta_{\mathrm{R}}},
$$

which is convenient to apply in the range $\omega_{\mathrm{H}} \ll \omega$ where, with very high accuracy, the term $g$ can be reduced to the electron term $g_{e}$ only, i.e. $g \simeq g_{\mathrm{e}}\left(1+o\left[(m / M)\left(\omega_{\mathrm{LH}}^{2} / \omega^{2}\right)\right]\right)$; thus one gets a reliable estimate in the form

$$
\frac{(\varepsilon-\chi)}{g} \simeq-\frac{\omega}{\omega_{\mathrm{c}}} \text {. }
$$

Actually, in overdense plasmas where $\omega_{\mathrm{pe}}^{2} / \omega_{\mathrm{c}}^{2} \gg 1$ (Wieder, $1964)$, i.e. $\mu \ll 1$, the polarization coefficient of the electron 
whistler waves is $\alpha_{x}=-\operatorname{sign}(\cos \theta)$ at $\omega \gg \omega_{\mathrm{LH}}$; thus the wave magnetic field polarization ellipse is very close to a circle. However, in the general case considered in Eq. (C3), one can obtain for low frequency whistlers near $\omega_{\mathrm{LH}}$ (where $\left.\chi=1-\omega_{p i}^{2} / \omega^{2}\right)$ that

$\frac{\alpha_{x}^{2}-1}{\alpha_{x}} \simeq \frac{\omega_{\mathrm{c}}}{\omega} \chi \frac{\sin ^{2} \theta}{\eta \cos \theta} \simeq-\frac{\omega}{\omega_{\mathrm{c}} \cos \theta}\left(1-\frac{\omega_{\mathrm{pi}}^{2}}{\omega^{2}}\right) \mu \sin ^{2} \theta$.

For the sheared whistler propagation regime, reminding that $\kappa=\widetilde{\kappa}(1+\mu)=\omega_{\text {pe }}^{2} / k^{2} c^{2}$, one gets for $\mu \widetilde{\kappa} \cos ^{2} \theta \ll 1$ the following relation (see also Lundin and Krafft, 2001)

$\cos ^{2} \theta=\frac{\omega^{2}}{\omega_{\mathrm{LH}}^{2}} \frac{\omega_{\mathrm{pi}}^{2}}{\omega_{\mathrm{pe}}^{2}}(1+\kappa)\left(1-\frac{\omega_{\mathrm{LH}}^{2}}{\omega^{2}}+\widetilde{\kappa}\right)$.

Then in the electron whistler quasi-resonant regime, Eq. (C7) with $\kappa, \widetilde{\kappa} \ll 1$ provides at $\omega=\omega_{\text {pi }}$ that $\cos ^{2} \theta=(1+$ $\mu)\left(\omega^{2} / \omega_{\mathrm{c}}^{2}\right)\left(1-\omega_{\mathrm{LH}}^{2} / \omega^{2}\right)=\omega_{\mathrm{pi}}^{2} / \omega_{\mathrm{c}}^{2} \ll 1$, so that

$\frac{\alpha_{x}^{2}-1}{\left|\alpha_{x}\right|} \simeq \frac{\omega_{\mathrm{pi}}}{\omega_{c}|\cos \theta|}\left(1-\frac{\omega_{\mathrm{pi}}^{2}}{\omega^{2}}\right) \mu=\left(1-\frac{\omega_{\mathrm{pi}}^{2}}{\omega^{2}}\right) \mu$.

The variation $\Delta\left|\alpha_{x}\right|=\left|\alpha_{x}\right|-1$ of the ratio of the polarization ellipse's axes can be estimated using the equation

$\frac{\Delta\left|\alpha_{x}\right|}{\alpha_{x}^{2}}\left(1+\alpha_{x}^{2}\right)=2 \mu \frac{\omega_{p i}^{2}}{\omega^{2}} \frac{\Delta \omega}{\omega}$,

with $\Delta \omega=\omega-\omega_{\mathrm{pi}}$, so that at $\omega=\omega_{\mathrm{pi}}$ where $\left|\alpha_{x}\right|=1$, one has

$\Delta\left|\alpha_{x}\right|=\mu \frac{\Delta \omega}{\omega}$.

Then, near $\omega_{\mathrm{pi}}$, the variation of $\left|\alpha_{x}\right|$ around unity will not be smooth if $\mu$ is not small - what is typical of the Earth magnetosphere at altitudes below $4000 \mathrm{~km}$ (Sonwalkar et al., 2004). Actually, the large axis of the magnetic field polarization ellipse performs a rotation of $\pi / 2$ when the frequency crosses the local ion plasma frequency $\omega_{\mathrm{pi}}$; the ellipse becomes a circle exactly at $\omega_{\mathrm{pi}}$.

The general case of the registration of non plane wave fields and the application of the spectral matrixes' technique to recover the ion plasma frequency will be considered in forthcoming papers.

\section{Appendix D}

\section{Averaged gyrofrequency and charge-to-mass ratio of the IPB, and their scattering}

To find the frequency interval where $\left|\left\langle\omega_{\mathrm{IPB}}\right\rangle\right|$ (Eq. 8) is localized, let us first prove that it is equivalent to the averaged gyrofrequency $\left\langle\omega_{\mathrm{b}}\right\rangle$ of the heavy plasma species

$\left\langle\omega_{\mathrm{b}}\right\rangle=\sum_{\mathrm{IPBj}}\left|\omega_{j}\right|\left\{\left|v_{j}\right| / \sum_{\text {IPBk }}\left|v_{k}\right|\right\}$, with

$$
\begin{aligned}
& \sum_{\operatorname{IPBk}}\left|v_{k}\right|=\sum_{\operatorname{IPB} \beta}\left|v_{\beta}\right|+\sum_{\operatorname{IPB} \alpha}\left|v_{\alpha}\right|=2-v_{\mathrm{e}}-\sum_{\gamma=1,2}\left|v_{\gamma}\right| \\
& =v_{\mathrm{e}}\left(\frac{2}{v_{\mathrm{e}}}-2+\frac{v_{\mathrm{b}}}{v_{\mathrm{e}}}\right),
\end{aligned}
$$

where the positive light ions are labeled with the indices $\gamma=1,2$. The summation over $\operatorname{IPB} \alpha($ resp. IPB $\beta$ ) is performed over the relative charge density of the positive (resp. negative) heavy species or IPB. Here the charge neutrality condition was used in the form

$$
\begin{aligned}
& 1=v_{1}+v_{2}+\sum_{\operatorname{IPB} \alpha}\left|v_{\alpha}\right|=v_{\mathrm{e}}+\sum_{\operatorname{IPB} \beta}\left|v_{\beta}\right|, \\
& v_{\mathrm{e}}=v_{1}+v_{2}+\sum_{\operatorname{IPB} \alpha}\left|v_{\alpha}\right|-\sum_{\operatorname{IPB} \beta}\left|v_{\beta}\right| \equiv v_{\mathrm{b}}+v_{1}+v_{2},
\end{aligned}
$$

where the resultant relative charge density $v_{\mathrm{b}}$ of the heavy background can be positive or negative. For $\nu_{\mathrm{b}}>0$ one has

$v_{\mathrm{e}}=1, \quad \frac{1}{2 / v_{\mathrm{e}}-2+v_{\mathrm{b}} / \nu_{\mathrm{e}}}=\frac{v_{\mathrm{e}}}{v_{\mathrm{b}}}=\frac{v_{\mathrm{e}}}{\left|v_{\mathrm{b}}\right|}$,

and for $\nu_{\mathrm{b}}<0$

$\frac{1}{v_{\mathrm{e}}}=1+\frac{\left|\nu_{\mathrm{b}}\right|}{v_{\mathrm{e}}}$,

$\frac{1}{2 / \nu_{\mathrm{e}}-2+v_{\mathrm{b}} / \nu_{\mathrm{e}}}=\frac{1}{2\left|\nu_{\mathrm{b}}\right| / \nu_{\mathrm{e}}+v_{\mathrm{b}} / \nu_{\mathrm{e}}}=\frac{\nu_{\mathrm{e}}}{\left|\nu_{\mathrm{b}}\right|}$.

Thus we proved that $\left|\left\langle\omega_{\mathrm{IPB}}\right\rangle\right|$, as defined by Eq. (8), is equal to $\left\langle\omega_{\mathrm{b}}\right\rangle$ and that, as a consequence, the so-called "averaged gyrofrequency" of the IPB lies between the minimum and the maximum values of the gyrofrequencies of all the varieties of heavy charged species. The corresponding averaged chargeto-mass ratio can be recovered directly from (D1) as

$$
\left\langle Z_{\mathrm{IPB}} M / M_{\mathrm{IPB}}\right\rangle=\left|\left\langle\omega_{\mathrm{IPB}}\right\rangle\right| / \omega_{\mathrm{H}} .
$$

Let us find the relative errors of the recovered parameters $\left\langle\omega_{\text {IPB }}\right\rangle$ and $\nu_{\mathrm{b}} / \nu_{\mathrm{e}}$ due to the scattering of (i) the cutoff frequencies $\left|\widehat{\omega}_{+}\right|$and $\left|\widehat{\omega}_{-}\right|$and (ii) the high frequencies $\omega_{\text {pi }}$ and $\omega_{\text {pe. }}$ Using Eqs. (14 )-(15), we can write that

$\frac{v_{\mathrm{b}}}{v_{\mathrm{e}}}\left\langle\omega_{\mathrm{IPB}}\right\rangle=\frac{\widehat{\omega}_{+} \widehat{\omega}_{-}}{\omega_{1} \omega_{2}}(L-S)$,

where

$$
\begin{aligned}
& \frac{v_{\mathrm{b}}}{v_{\mathrm{e}}}=1-\frac{\nu_{1}}{v_{\mathrm{e}}}-\frac{\nu_{2}}{v_{\mathrm{e}}}=\frac{\widehat{\omega}_{+} \widehat{\omega}_{-}}{\omega_{1} \omega_{2}} \\
& +\frac{\left(\omega_{1}+\omega_{2}\right)}{\omega_{1} \omega_{2}}(L-S) \frac{\widehat{\omega}_{+} \widehat{\omega}_{-}}{\omega_{1} \omega_{2}}+\frac{\left(\widehat{\omega}_{+}+\widehat{\omega}_{-}\right)}{\omega_{1} \omega_{2}}(L-S),
\end{aligned}
$$

where $L$ and $S$ are defined by Eqs. (14) and (15). 
Then, in the case (i), considering the scattering $\Delta \widehat{\omega}_{ \pm}$of the measured cutoff frequencies, we get from Eq. (D8) that

$$
\begin{aligned}
& -\Delta\left(\frac{\nu_{\mathrm{b}}}{v_{\mathrm{e}}}\right)=\Delta\left(\frac{\nu_{1}}{v_{\mathrm{e}}}\right)+\Delta\left(\frac{\nu_{2}}{v_{\mathrm{e}}}\right)= \\
& -\Delta\left(\widehat{\omega}_{+} \widehat{\omega}_{-}\right)\left[\frac{1}{\omega_{1} \omega_{2}}+\frac{\omega_{1}+\omega_{2}}{\omega_{1}^{2} \omega_{2}^{2}}(L-S)\right] \\
& -\Delta\left(\widehat{\omega}_{+}+\widehat{\omega}_{-}\right) \frac{1}{\omega_{1} \omega_{2}}\left[L-S-\left(\widehat{\omega}_{+}+\widehat{\omega}_{-}\right)\right] \\
& +\Delta\left(\widehat{\omega}_{+}+\widehat{\omega}_{-}\right) \frac{\left(\omega_{1}+\omega_{2}\right)}{\omega_{1} \omega_{2}} \frac{\widehat{\omega}_{+}}{\omega_{1} \widehat{\omega}_{-}},
\end{aligned}
$$

and from Eq. (D7) that

$$
\begin{aligned}
& \Delta\left(\frac{\nu_{\mathrm{b}}}{v_{\mathrm{e}}}\right)\left\langle\omega_{\mathrm{IPB}}\right\rangle+\frac{\nu_{\mathrm{b}}}{v_{\mathrm{e}}} \Delta\left\langle\omega_{\mathrm{IPB}}\right\rangle= \\
& \left(\frac{\Delta \widehat{\omega}_{+}}{\widehat{\omega}_{+}}+\frac{\Delta \widehat{\omega}_{-}}{\widehat{\omega}_{-}}\right) \frac{\nu_{\mathrm{b}}}{\nu_{\mathrm{e}}}\left\langle\omega_{\mathrm{IPB}}\right\rangle-\Delta\left(\widehat{\omega}_{+}+\widehat{\omega}_{-}\right) \frac{\widehat{\omega}_{+} \widehat{\omega}_{-}}{\omega_{1} \omega_{2}},
\end{aligned}
$$

so that the relative error of the averaged gyrofrequency of the IPB due to the scattering of the ion cutoff frequencies is

$$
\begin{aligned}
& \frac{\Delta\left\langle\omega_{\mathrm{IPB}}\right\rangle}{\left\langle\omega_{\mathrm{IPB}}\right\rangle}= \\
& \left(\frac{\Delta \widehat{\omega}_{+}}{\widehat{\omega}_{+}}+\frac{\Delta \widehat{\omega}_{-}}{\widehat{\omega}_{-}}\right)-\frac{\Delta\left(\widehat{\omega}_{+}+\widehat{\omega}_{-}\right)}{(L-S)}-\Delta\left(\frac{\nu_{\mathrm{b}}}{\nu_{\mathrm{e}}}\right) /\left(\frac{\nu_{\mathrm{b}}}{v_{\mathrm{e}}}\right) .
\end{aligned}
$$

In the case (ii), considering the scattering $\Delta \omega_{\text {pi }}$ and $\Delta \omega_{\text {pe }}$ of the measured plasma frequencies, we get from Eqs. (D7)(D8) and (14) that

$$
\begin{aligned}
& \frac{\Delta L}{L}=\left(\frac{\Delta \omega_{\mathrm{pi}}^{2}}{\omega_{\mathrm{pi}}^{2}}-\frac{\Delta \omega_{\mathrm{pe}}^{2}}{\omega_{\mathrm{pe}}^{2}}\right), \\
& \Delta\left(\frac{\nu_{\mathrm{b}}}{\nu_{\mathrm{e}}}\right)\left\langle\omega_{\mathrm{IPB}}\right\rangle+\frac{\nu_{\mathrm{b}}}{\nu_{\mathrm{e}}} \Delta\left\langle\omega_{\mathrm{IPB}}\right\rangle=\frac{\widehat{\omega}_{+} \widehat{\omega}_{-}}{\omega_{1} \omega_{2}} \Delta L, \\
& \Delta\left(\frac{\nu_{\mathrm{b}}}{\nu_{\mathrm{e}}}\right)=\omega_{\mathrm{c}} \frac{\omega_{\mathrm{pi}}^{2}}{\omega_{\mathrm{pe}}^{2}}\left(\frac{\Delta \omega_{\mathrm{pi}}^{2}}{\omega_{\mathrm{pi}}^{2}}-\frac{\Delta \omega_{\mathrm{pe}}^{2}}{\omega_{\mathrm{pe}}^{2}}\right) \\
& \times \frac{\left(\widehat{\omega}_{+}+\widehat{\omega}_{-}\right)+\left(\omega_{2}+\omega_{1}\right)\left(\widehat{\omega}_{+} \widehat{\omega}_{-} / \omega_{1} \omega_{2}\right)}{\omega_{2} \omega_{1}},
\end{aligned}
$$

so that the relative error of the averaged gyrofrequency of the IPB due to the scattering of the ion and electron plasma frequencies is

$$
\begin{aligned}
& \frac{\Delta\left\langle\omega_{\mathrm{IPB}}\right\rangle}{\left\langle\omega_{\mathrm{IPB}}\right\rangle}= \\
& \frac{\omega_{\mathrm{c}}}{L-S} \frac{\omega_{\mathrm{pi}}^{2}}{\omega_{\mathrm{pe}}^{2}}\left(\frac{\Delta \omega_{\mathrm{pi}}^{2}}{\omega_{\mathrm{pi}}^{2}}-\frac{\Delta \omega_{\mathrm{pe}}^{2}}{\omega_{\mathrm{pe}}^{2}}\right)-\Delta\left(\frac{\nu_{\mathrm{b}}}{\nu_{\mathrm{e}}}\right) /\left(\frac{\nu_{\mathrm{b}}}{\nu_{\mathrm{e}}}\right) .
\end{aligned}
$$

For the numerical parameters of Figs. 1-2 one finds that, in the selected domain of altitudes, the values of $\Delta\left\langle\omega_{\mathrm{IPB}}\right\rangle$ and of the variation $\Delta \omega_{\text {cut1 }}$ of the ion cutoff frequency of the electron whistlers are of the same order, i.e. $\Delta\left\langle\omega_{\mathrm{IPB}}\right\rangle \sim$ $(1 \div 2) \Delta \omega_{\text {cut } 1}$. The regular distorsion of the recovered value of $\left\langle\omega_{\mathrm{IPB}}\right\rangle$ from the actual gyrofrequency of the contaminating heavy ions, especially in the low altitude domain of Figs. 12 , is connected with the validity conditions (24), which are not satisfied with large excess at low altitudes and for small levels of contamination, i.e. small $\left|\nu_{\mathrm{b}}\right| / \nu_{\mathrm{e}}$.

Acknowledgements. This work was supported by the Centre National de la Recherche Scientifique (CNRS, France) and the Russian Academy of Sciences in the frame of the International Scientific Collaboration Project PICS No. 4960 and of the Grant RFBR 09-02-91052-CNRS_ $a$. The authors acknowledge the Institut National des Sciences de l'Univers (INSU, France).

Topical Editor I. A. Daglis thanks two anonymous referees for their help in evaluating this paper.

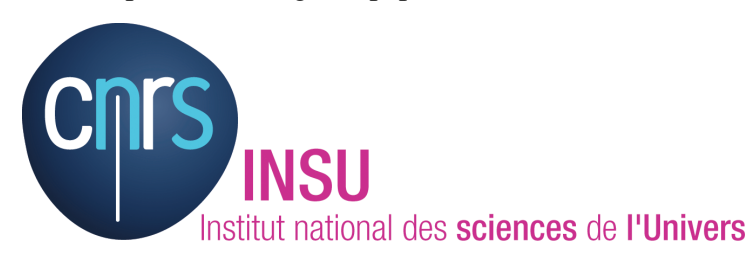

The publication of this article is financed by CNRS-INSU.

\section{References}

Angerami, J. J. and Thomas, J. O.: Studies of planetary atmospheres, 1. The distribution of electrons and ions in the Earth's exosphere, J. Geophys. Res., 69(21), 4537-4560, 1964.

Brice, N. M. and Smith, R. L.: Lower hybrid resonance emissions, J. Geophys. Res., 70(1), 71-80, 1965.

Buchsbaum, S. J.: Resonance in a plasma with two ion species, Phys. Fluids, 3(3), 418-420, 1960.

Coates, A. J., Wellbrock, A., Lewis, G. R., Jones, G. H., Young, D. T., Crary, F. J., and Waite Jr., J. H.: Heavy negative ions in Titan's ionosphere: Altitude and latitude dependance, Planet. Space Sci., 57, 1866-1871, 2009.

Cravens, T. E., Robertson, I. P., Waite Jr., J. H., Yelle, R. V., Kasprzak, W. T., Keller, C. N., Ledvina, S. A., Niemann, H. B., Luhmann, J. G., McNutt, R. L., Ip, W.-H., De La Haye, V., Mueller-Wodarg, I., Wahlund, J.-E., Anicich, V. G., and Vuitton, V.: Composition of Titan's ionosphere, Geophys. Res. Lett., 33, L07105, doi:10.1029/2005GL025575, 2006.

Cravens, T. E., McNudt Jr., R. L., Waite Jr., J. H., Robertson, I. P., Lukhmann, J. G., Kasprzak, W., and Ip, W.-H.: Plume ionosphere of Enceladus as seen by the Cassini ion and neutral mass-spectrometer, Geophys. Res. Lett., 36, L08106, doi:10.1029/2008GLO37811, 2009.

Das, G. C. and Uberoi, C.: Crossover frequencies in multicomponent plasmas with negative ions, J. Geophys. Res., 77(28), 5597$5601,1972$.

Gintzburg, M. A.: Low-frequency waves in multicomponent plasma, Geomagnetism Aeronomy, 3, 610-614, 1963. 
Gurnett, D. A. and Bhattacharjee, A.: Introduction to plasma physics: with space and laboratory applications, Cambridge University Press, Cambridge, UK, 2005.

Gurnett, D. A. and Burns, T. B.: The low-frequency cutoff of ELF emissions, J. Geophys. Res., 73(23), 7437-7445, 1968.

Helliwell, R. A.: Whistlers and Related Ionospheric Phenomena, Stanford University Press, Stanford, Calif., 1965.

Hines, C. O.: Heavy-ion effects in audio-frequency radio propagation, J. Atmos. Terr. Phys., 11, 36-42, 1957.

Krafft, C. and Volokitin, A.: Interaction of suprathermal solar wind electron fluxes with sheared whistler waves: fan instability, Ann. Geophys., 21, 1393-1403, doi:10.5194/angeo-21-13932003, 2003.

Krafft, C., Volokitin, A., and Zaslavsky, A.: Saturation of the fan instability: nonlinear merging of resonances, Phys. Plasmas, 12, 112309, doi:10.1063/1.2118727, 2005.

Krafft, C. and Volokitin, A.: Stabilization of the fan instability: Electron flux relaxation, Phys. Plasmas, 13, 122301, doi:10.1063/1.2372464, 2006.

Lundin, B. V. and Krafft, C.: On the focusing of whistler wave field intensity in a quasi-resonant propagation regime, J. Geophys. Res., 102(A1), 141-150, 1997.

Lundin, B. V. and Krafft, C.: On the similarity features of normalized frequency spectra of magnetospherically reflected whistlers, J. Geophys. Res., 106(A11), 25643-25654, 2001.

Lundin, B. V. and Krafft, C.: Modified electron whistler dispersion law, J. Plasma Phys., 67(2-3), 149-161, 2002.

Lundin, B. V. and Krafft, C.: On the dispersion law of lowfrequency electron whistler waves in a multi-ion plasma, Ann. Geophys., 26, 1605-1615, doi:10.5194/angeo-26-1605-2008, 2008.

Lundin, B. V. and Krafft, C.: On the electron whistler dispersion law in a cold plasma with light ions and heavy charged particulates, Phys. Plasmas, 16, 052104, 1-11, 2009a.

Lundin, B. V. and Krafft, C.: On the minimal set of plasma parameters to determine the dispersion law of electron whistler waves, Plasma Phys. Rep., 35(6), 502-509, 2009b.

Mann, I., Murad, E., and Czechowski, A.: Nanoparticles in the inner solar system, Planet. Space Sci., 55, 1000-1009, 2007.

Meyer-Vernet, N., Lecacheux, A., Kaiser, M. L., and Gurnett, D. A.: Detecting nanoparticles at radio frequencies: Jovian dust streams impacts on Cassini/RPWS, Geophys. Res. Lett., 36, L03103, doi:10.1029/2008GLO36752, 2009.

Meyer-Vernet, N., Czechowski, A., Mann, I., Maksimovic, M., Lecacheux, A., Goetz, K., Kaiser, M. L., St.Cyr, O. C., BaIe, S. D., and Le Chat, G.: Detection of fast nanoparticles in the solar wind, AIP Conference Proceedings, vol. 1216, pp. 502-505, XII International Solar Wind Conference, doi:10.1063/1.3395912, 2010.

Muzzio, J. L. R.: Ion cutoff whistlers, J. Geophys. Res., 73(23), 7526-7529, 1968.

Rauch, J. L., Trotignon, J. G., Cornilleau-Wehrlin, N., Robert, P., and Titotva, E.: Determination inside the plasmasphere of the ion composition and absolute density from the ULF wave propagation properties with WHISPER/CLUSTER and STAFF/CLUSTER data, Abstract of report on the 4th VERSIM Workshop, 13-17 September, Prague, Czech Republic, 2010.
Shafranov, V. D.: Electromagnetic Waves in Plasma, in Review of Plasma Physics, Consultants Bureau, N.Y., v. 3, pp. 1-157, 1967.

Shawhan, S. D.: Experimental Observations of Proton Whistlers from Injun 3 VLF Data, J. Geophys. Res., 71(1), 29-45, 1966.

Shawhan, S. D. and Gurnett, D. A.: Fractional Concentration of Hydrogen Ions in the Ionosphere from VLF Proton Whistler Measurement, J. Geophys. Res., 71(1), 47-59, 1966.

Shawhan, S. D.: Negative Ion Detection in the Ionosphere from Effects on ELF Waves, J. Geophys. Res., 71(23), 5585-5598, 1966.

Sittler Jr., E. C., Ali, A., Cooper, J. F., Hartle, R. E., Johnson, R. E., Coates, A. J., and Young, D. T.: Heavy ion formation in Titan's ionosphere: magnetospheric introduction of free oxygen and a source of Titan's aerosols?, Plan. Space Sci., 57, 1547-1557, 2009.

Smith, J.: Negative ion effects on whistler mode propagation, J. Geophys. Res., 70(1), 53-59, 1965.

Smith, R. L. and Brice, N. M.: Propagation in a multicomponent plasma, J. Geophys. Res., 69(23), 5029-5040, 1964.

Sonwalkar, V. S., Carpenter, D. L., Bell, T. F., Spasojevic', M., Inan, U. S., Li, J., Chen, X., Venkatasubramanian, A., Harikumar, J., Benson, R. F., Taylor, W. W. L., and Reinisch, B. W.: Diagnostics of magnetospheric density and irregularities at altitudes $5000 \mathrm{~km}$ using whistler and $\mathrm{Z}$ mode echoes from radio sounding on the IMAGE satellite, J. Geophys. Res., 109, A11212, 1-22, 2004.

Volokitin, A. and Krafft, C.: Interaction of suprathermal electron fluxes with lower hybrid waves, Phys. Plasmas, 11, 3165-3176, 2004.

Wahlund, J.-E., Galand, M., Muller-Wodarg, I., Cui, J., Yelle, R. V., Crary, F. J., Mandt, K., Magee, B., Waite Jr., J. H., Young, D. T., Coates, A. J., Garnier, P., Agren, K., André, M., Eriksson, A. I., Cravens, T. E., Vuitton, V., Gurnett, D. A., and Kurth, W. S.: On the amount of heavy molecular ions in Titan's ionosphere, Planet. Space Sci., 57, 1857-1865, 2009.

Wieder, B.: Microwave propagation in an overdense bounded magnetosplasma, Phys. Fluids, 7(7), 964-972, 1964.

Yakimenko, V. L.: Oscillations in a cold plasma containing two ion species, Tech. Phys., 7(2), 117-124, 1962.

Young, D. T., Berthelier, J.-J., Blanc, M., Burch, J. L., Bolton, S., Coates, A. J., Crary, F. J., Goldstein, R., Grande, M., Hill, T. W., Johnson, R. E., Baragiola, R. A., Kelha, V., McComas, D. J.,Mursula, K., Sittler,E. C., Svenes, K. R., Szegö, K., Tanskanen, P., Thomsen, M. F., Bakshi, S. , Barraclough, B. L., Bebesi, Z., Delapp, D., Dunlop, M. W., Gosling, J. T., Furman, J. D.,Gilbert, L. K., Glenn, D., Holmlund, C., Illiano, J.-M., Lewis, G. R., Linder, D. R., Maurice, S. McAndrews, H. J. Narheim, B. T., Pallier, E., Reisenfeld, D., Rymer, A. M., Smith, H. T., Tokar, R. L., Vilppola, J., and Zinsmeyer, C.: Composition and Dynamics of Plasma in Saturn's Magnetosphere, Science, 307, 1262-1266, 2005. 